\title{
Relaxation procedures for solving Signorini-Coulomb contact problems
}

\author{
P. Bisegna ${ }^{\mathrm{a}}$, F. Lebon ${ }^{\mathrm{b}, *}$, F. Maceri ${ }^{\mathrm{a}}$ \\ a Dipartimento di Ingegneria Civile, Universitadi Roma 'Tor Vergata', 00133 Roma, Italy \\ Laboratoire de Mécanique et d'Acoustique and Université de Provence, 31, Ch. Joseph Aiguier, 13402 Marseille Cedex 20, France
}

\begin{abstract}
This paper deals with the numerical solution of two-dimensional unilateral contact problems with friction between a linearly elastic body and a rigid obstacle. The contact is modeled by Signorini's law and the friction by Coulomb's law. A discrete dual formulation condensed on the contact zone is introduced and the contact forces are obtained either by relaxation or by block-relaxation procedures. A comparison is presented between these two techniques.
\end{abstract}

\section{Introduction}

The aim of this paper is to present numerical methods to solve unilateral contact discrete plane problems with dry friction. Unilateral contact and friction are respectively modeled by Signorini's and Coulomb's laws, which constitute a simple and useful framework for the analysis of unilateral frictional contact problems of a linearly elastic body with a rigid support [5]. It seems useful, from a mechanical point of view, to develop techniques based on dual formulations, in order to directly compute stresses, which are the quantities of primary interest. The continuous and discrete dual formulations of the contact problem lead to quasi-variational inequalities, whose unknowns, after condensation, are the normal and tangential contact forces at points/nodes of the initial contact area [1,3,4,6-8]. New numerical solution methods, based on iterative relaxation and block-relaxation techniques, are proposed $[4,9]$. The relaxation procedure is a succession of local minimizations in given convexes. The definition of the convex of constraints, which is a cylinder, varies for tangential or normal components. This algorithm turns out to be very robust. At the typical step of the block-relaxation iteration, two sub-problems are solved one after the other: the former is a problem of friction with given normal forces, and the latter is a problem of unilateral contact with prescribed

\footnotetext{
* Corresponding author.
}

tangential forces. Both of them are standard problems of quadratic programming. This method is the dual version of the famous PANA algorithm [2], and, for sufficiently small friction coefficients, the typical step of the block iteration is a contraction [4]. The contraction principle implies the wellposedness of the discrete dual condensed formulation, the convergence of the proposed algorithm, and an estimate of the convergence rate. Both these procedures are applied here to various examples, in the case of elastic and piezoelectric bodies in contact with rigid foundations [3,4], in order to evaluate their efficiency and robustness.

\section{Formulation of the problem}

\subsection{Notations and strong formulation}

Let $R^{3}$ be the Euclidian point space, and $\left(O, x_{1}, x_{2}, x_{3}\right)$ a Cartesian frame whose unit vectors are $e_{1}, e_{2}, e_{3}$. Cartesian components are denoted by subscript indices. Einstein's summation convention is adopted. The inner and vector products are denoted by the symbols (.) and ( $X)$, respectively. The length of a vector is denoted by $|\cdot|$. Differentiation with respect to $x_{i}$ is denoted by $(\cdot)_{i}$. Let the regular bounded region $\Omega \subset R^{3}$ be the reference configuration of a deformable body, whose boundary is denoted by $\Gamma$, and let $n$ be the outward normal unit vector to $\Gamma$. The body is subjected to volume forces $F$ and to surface 
forces $f$ on $\Gamma_{f} \subset \Gamma$. On $\Gamma_{d} \subset \Gamma / \Gamma_{f}$ the displacement $u_{o}$ is given. The remaining part $\Gamma_{c}=\Gamma /\left(\Gamma_{f} \cup \Gamma_{d}\right)$ of the boundary $\Gamma$ is in receding contact with a rigid support, modelled according to the Signorini unilateral contact law and the Coulomb dry friction law. The body is comprised of a linearly elastic material, whose elasticity $A$ and compliance $S$ fourth-order tensors are assumed to be positive definite and uniformly bounded in $\Omega$. The contact problem is studied in the framework of the quasi-static small deformation theory, under monotonic loads. The unknowns are the displacement field $u$ and the stress field $\sigma$ in $\Omega$, and the governing equations are

$\sigma_{i j, j}+F_{i}=0$ in $\Omega, \quad \sigma_{i j}=\sigma_{j i}$ in $\Omega$,

$\sigma_{i j}=A_{i j k l} \epsilon_{k l}(u)$ in $\Omega, \quad \epsilon_{i j}(u)=\frac{1}{2}\left(u_{i, j}+u_{j, i}\right)$ in $\Omega$,

$\sigma_{i j} n_{j}=f_{i}$ on $\Gamma_{f}, \quad u=u_{o}$ on $\Gamma_{d}$,

$\sigma_{N} \leq 0, u_{N} \leq 0, u_{N} \sigma_{N}=0$ on $\Gamma_{c}$,

$\left|\sigma_{T}\right| \leq-\mu \sigma_{N}, \quad \exists \lambda \geq 0: u_{T}$

$$
=-\lambda \sigma_{T},\left(\left|\sigma_{T}\right|+\mu \sigma_{N}\right) u_{T}=0 \text { on } \Gamma_{c},
$$

where $\mu \geq 0$ is the friction coefficient and the normal and tangential displacement and traction components are, as usual, defined by

$\sigma_{N}=\sigma n \cdot n, \quad \sigma_{T}=\sigma n-\sigma_{N} n, \quad u_{N}=u \cdot n$,

$u_{T}=u-u_{N} n$

\subsection{Variational formulations}

\subsubsection{Primal formulation}

The primal variational formulation of the contact problem consists of the implicit variational inequality:

Find $u \in \mathscr{K}_{d}$ such that $\forall v \in \mathscr{K}_{d}$

$a(u, v-u)+j(u, v)-j(u, u)-L(v-u) \geq 0$,

where

$a(u, v)=\int_{\Omega} A_{i j k l} \epsilon_{i j}(u) \epsilon_{k l}(v) \mathrm{d} \Omega$,

$L(v)=\int_{\Omega} F_{i} v_{i} \mathrm{~d} \Omega+\int_{\Gamma_{f}} f_{i} v_{i} \mathrm{~d} \Gamma$,

$j(u, v)=-\int_{\Gamma_{c}} \mu \sigma_{N}(u)\left|v_{T}\right| \mathrm{d} \Gamma$.

and where

$V=\left\{v \in\left(H^{1}(\Omega)\right)^{3}, \gamma v=u_{o}\right.$ on $\left.\Gamma_{d}\right\}$,

$\mathscr{K}_{d}=\left\{v \in V, v_{N} \leq 0\right.$ on $\left.\Gamma_{c}\right\}$.
Here $\mathscr{K}_{d}$ is the convex set of kinematically admissible fields and $\gamma$ is the trace operator on the boundary $\Gamma$ from $\left(H^{1}(\Omega)\right)^{3}$ to $\left(H^{(1 / 2)}(\Gamma)\right)^{3}$. Moreover, $A$ is positive definite, $A \in L^{\infty}(\Omega)$, $\mu \in L^{\infty}\left(\Gamma_{c}\right), \mu \geq 0, F \in\left(L^{2}(\Omega)\right)^{3}$ and $f \in\left(L^{2}(\Gamma)\right)^{3}$. Classically, this problem is equivalent to a fixed point problem coupled with a convex and non-differentiable minimization problem [1]. Existence and uniqueness results are available for small friction coefficients [11].

\subsubsection{Dual formulation}

The dual formulation of the contact problem consists of the quasi-variational inequality [6]:

Find $\sigma \in \mathscr{K}_{C\left(-\sigma_{N}\right)}$ such that $\forall \tau \in \mathscr{K}_{C\left(-\sigma_{N}\right)}$

$b(\sigma, \tau-\sigma)-l(\tau-\sigma) \geq 0$,

where

$b(\sigma, \tau)=\int_{\Omega} S_{i j k l} \sigma_{i j} \tau_{k l} \mathrm{~d} \Omega, \quad l(\tau)=\int_{\Gamma_{d}} \tau_{i j} n_{j} u_{o i} \mathrm{~d} \Gamma$,

and where

$H=\left\{\tau \in L^{2}(\Omega, M) ; \tau_{i j, j}+F_{i}=0\right.$ in $\Omega ; \tau n=f$ on $\left.\Gamma_{f}\right\}$

$\mathscr{K}_{C(\eta)}=\left\{\tau \in H ; \tau_{N} \leq 0\right.$ on $\Gamma_{c} ;\left|\tau_{T}\right| \leq \mu \eta$ on $\left.\Gamma_{c}\right\}$.

Here $M$ is the space of second order symmetric tensors and $\mathscr{K}_{C\left(-\sigma_{N}\right)}$, the convex set of statically admissible stress fields, depends on the solution $\sigma$. Moreover, $S$ is positive definite, $S_{i j k l} \in L^{\infty}(\Omega), \quad 1 \leq i, j, k, l \leq 3, \mu \in$ $L^{\infty}\left(\Gamma_{c}\right), \mu \geq 0, u_{o} \in\left(H^{(1 / 2)}(\Gamma)\right)^{3}$. In the following, $\mathscr{K}_{\theta}$ the local convex subset of $\left(H^{-(1 / 2)}\left(\Gamma_{c}\right)\right)^{3}$ is introduced

$\mathscr{K}_{\theta}=\left\{\tau \in\left(H^{-(1 / 2)}\left(\Gamma_{c}\right)\right)^{3} ; \tau_{N} \leq 0 ;\left|\tau_{T}\right| \leq \mu \theta\right\}$.

\section{Condensed dual formulation}

\subsection{Continuous formulation}

The aim of this section is to present the dual formulation condensed on the contact zone. This formulation, etablished by Telega [6], is based on the Mosco duality ([12], see also Ref. [13]). The primal formulation is re-written in the following form

Find $u \in V$ such that $\forall v \in V$

$$
\begin{aligned}
& a(u, u)+I_{K_{d}}(u)+j(u, u)-L(u) \\
& \quad \leq a(u, v)+I_{K_{d}}(v)+j(u, v)-L(v),
\end{aligned}
$$

where $I_{K_{d}}$ is the indicator function of the set $K_{d}$. In the following, we define the two functionals $g$ and $\phi$ by

$g(v, w)=a(v, w)-L(w), \quad \phi(v, w)=I_{K_{d}}(w)+j(v, w)$.

The problem is then written

Find $u \in V$ such that $\forall v \in V$

$g(u, u)+\phi(u, u) \leq g(u, v)+\phi(u, v)$. 
With a slight abuse of notation, the functional $\phi$ is regarded as defined on $\left(H^{-(1 / 2)}\left(\Gamma_{c}\right)\right)^{2}$. We denote by $\phi^{*}$ the Fenchel conjugate of the functional $\phi$ with respect to the second variable, $\quad \phi^{*}\left(v,-w^{*}\right)=\sup \left\{\left\langle-w^{*}, w\right\rangle-\phi(v, w), w \in\right.$ $\left.H^{(1 / 2)}\left(\Gamma_{c}\right)\right\}$. Here $\langle\cdot, \cdot\rangle$ is the duality pairing between $H^{-(1 / 2)}\left(\Gamma_{c}\right)$ and $H^{(1 / 2)}\left(\Gamma_{c}\right)$. By definition of $\phi$, we obtain

$$
\begin{aligned}
\phi^{*}\left(v,-w^{*}\right)= & \sup \left\{\left\langle-w_{N}^{*}, w_{N}\right\rangle-I_{K_{d}}(w)\right\} \\
& +\sup \left\{\left\langle-w_{T}^{*}, w_{T}\right\rangle-j(v, w)\right\}=I_{K_{\sigma_{N}(v)}}\left(w^{*}\right)
\end{aligned}
$$

In the Mosco duality theory, it is assumed that

(A) $V$ is a real reflexive space and $V^{*}$ its dual space,

(B) $\forall v \in V, w \rightarrow \phi(v, w)$ is a real valued proper convex function on $V$,

(C) $\forall v \in V, w \rightarrow g(v, w)$ is a real valued convex function on $V$, which is continuous when $w=v$,

(D) $\forall v \in V, w \rightarrow g(v, w)$ has a Gateaux derivative with respect to $w, D g(v, w)$, in $w=v$, such that $\forall w^{*} \in V^{*}$ the set $\left\{v \in V ; D g(v, v)=w^{*}\right\}$ contains at most one element denoted $(D g)^{-1}\left(w^{*}\right)$.

Then the dual problem of Eq. (12) is [15]

Find $u^{*} \in V^{*}$ such that $\forall w^{*} \in V^{*}$

$$
\begin{aligned}
& \phi^{*}\left((D g)^{-1}\left(-u^{*}\right), u^{*}\right)-\left\langle u^{*},(D g)^{-1}\left(-u^{*}\right)\right\rangle \\
& \quad \leq \phi^{*}\left((D g)^{-1}\left(-u^{*}\right), w^{*}\right)-\left\langle w^{*},(D g)^{-1}\left(-u^{*}\right)\right\rangle
\end{aligned}
$$

In the present case

$g(v, w)=a(v, w)-L(w)=\langle A v-f, w\rangle$

$D g(u, u)=A u-f$

Thus,

$(D g)^{-1}\left(u^{*}\right)=u=A^{-1} u^{*}+A^{-1} f$

Let $A^{-1} u^{*}+A^{-1} f=G u^{*}+g, G$ is the Green's operator for the boundary value problem of linear elasticity. The dual problem is written

Find $u^{*}=(N, T) \in \mathscr{K}_{-N}$ such that $\forall w^{*} \in \mathscr{K}_{-N}$

$\left\langle w^{*}-u^{*}, G u^{*}+g\right\rangle \geq 0$.

where $N$ and $T$ are the normal and tangential components of the contact force $u^{*}$. It is emphasized that Eq. (17) involves only unknowns defined on the contact boundary. Hence, it can be regarded as the condensed version of the dual problem defined in Section 2. It is proved in Ref. [14], that $u$ is a solution of the primal problem if and only if $u^{*}$ is a solution of the condensed dual problem. Thus, in the following, we focus on the numerical solution of the condensed dual problem.

\subsection{Discretized formulation}

The discretization of Eq. (17) requires the internal discretization of the cone $\mathscr{K}_{-N}$ and the construction of an approximation of the Green's operator $G$. These tasks can be achieved by building a triangulation $T_{h}$ of the domain $\Omega$ consistant with the partition of boundary in $\Gamma_{c}, \Gamma_{f}$ and $\Gamma_{d}$ and by approximating the displacement field in piecewisepolynomial functions. In other words, an approximation of the Green's operator can be built from the inversion of the condensed (on the contact boundary) stiffness matrix. The discretized formulation, using standard notations, is written as:

Find $u^{*} \in \mathscr{K}_{-N}^{h}$ such that $\forall w^{*} \in \mathscr{K}_{-N}^{h}$

$\left\langle w^{*}-u^{*}, G_{h} u^{*}+g_{h}\right\rangle \geq 0$.

An alternative method useful to obtain a discretized version of Eq. (17), i.e. Eq. (18) could be based on the discretization of the dual formulation followed by the condensation on the contact boundary. From a numerical point of view, in this variant, is less expensive to build the stiffness matrix.

\section{Numerical procedures}

\subsection{First method: D-PANA algorithm}

In this section, a numerical algorithm to solve, in the plane case, the condensed dual problem is presented. The idea of the algorithm, based on a block-relaxation technique, consists in a sequence of solutions of two subproblems: at the $k$-th step, a problem of friction with given normal forces is solved, and then a problem of unilateral contact with prescribed tangential forces is solved, too. This algorithm is named D-PANA, since it is the dual version of the PANA classical algorithm due to P.D. Panagiotopoulos [2]. The symmetric positive definite compliance matrix $G_{h}$ is decomposed in four blocks as follows

$\left.G_{h}=\begin{array}{cc}G^{N N} & \left(G^{T N}\right)^{t} \\ G^{T N} & G^{T T}\end{array}\right)$,

where $G^{N N}$ (resp. $G^{T T}$ ) is the sub-matrix corresponding to the normal (resp. tangential) degrees of freedom. Analogously, the generalized displacement vector $g_{h}$ is decomposed in two blocks

$g_{h}=\left(\begin{array}{c}g^{N} \\ g^{T}\end{array}\right)$,

where $g^{N}$ (resp. $g^{T}$ ) corresponds to the normal (resp. tangential) degrees of freedom.

Let $\left(N^{0}, T^{0}\right)$ be given, the $k$-th iteration of the algorithm is,

- $T^{k+1}$ is the solution of the following friction problem with given normal contact force

$$
\min \left\{\frac{1}{2} T^{t} G^{T T} T+T^{t}\left(g^{T}+G^{T N} N^{k}\right), \quad|T| \leq-\mu N^{k}\right\}
$$


- $N^{k+1}$ is the solution of the following unilateral contact problem with given tangential friction force

$$
\min \left\{\frac{1}{2} N^{t} G^{N N} N+N^{t}\left(g^{N}+\left(G^{T N}\right)^{t} T^{k+1}\right), N \leq 0\right\}
$$

This algorithm is based on a simple idea: if in Eq. (18), friction forces (resp. contact forces) are given, the convex is fixed and the problem is equivalent to a minimization problem on the contact forces (resp. friction forces). It is noted that minimization problems (21) and (22) have unique solutions, since the involved functionals are strictly convex, and the minimization sets are convex and not empty. Moreover, these problems can be solved by efficient quadratic programming techniques [15] or relaxation algorithms [16]. It is possible to prove the following result

Theorem 1. For a sufficiently small friction coefficient $\mu$ the D-PANA algorithm is convergent.

The proof can be found in Ref. [4].

\subsection{Second method: D-GLT algorithm}

This algorithm is an adaptation of the algorithm proposed in Ref. [16] (see also Refs. [5,10]). The idea is to minimize a succession of one-dimensional problems, that is, for the $i$ thcomponent, the other components are fixed and we have to minimize a convex one-dimensional problem in a convex subset

- $N_{i} \leq 0 \forall \mathrm{i} \in\{1 \ldots \mathrm{n}\}$, for a normal contact component,

- $\left|T_{i}\right| \leq-\mu N_{i} \forall i \in\{1 \ldots n\}$, for a friction component.

The algorithm is given by

$\left(N^{0}, T^{0}\right)$ initial solution be given, for any $k>0$ (iteration index) and for $i \in\{1 \ldots n\}$ :

$$
\begin{aligned}
& \alpha=-g_{i}^{N}-\sum_{j=1}^{i-1} G_{i j}^{N N} N_{j}^{k+1}-\sum_{j=i+1}^{n} G_{i j}^{N N} N_{j}^{k}-\sum_{j=1}^{i-1} G_{i j}^{T T} T_{j}^{k+1} \\
& -\sum_{j=i}^{n} G_{i j}^{T T} T_{j}^{k} \\
& N_{i}^{k+(1 / 2)}=\frac{\alpha}{G_{i i}^{N N}} \\
& \begin{cases}\text { if } N_{i}^{k+(1 / 2)}>0 & \text { then } N_{i}^{k+1}=0 \\
\text { if } N_{i}^{k+(1 / 2)} \leq 0 & \text { then } N_{i}^{k+1}=N_{i}^{k+(1 / 2)}\end{cases} \\
& \beta=-g_{i}^{T}-\sum_{j=1}^{i} G_{i j}^{N N} N_{j}^{k+1}-\sum_{j=i+1}^{n} G_{i j}^{N N} N_{j}^{k}-\sum_{j=1}^{i-1} G_{i j}^{T T} T_{j}^{k+1} \\
& -\sum_{j=i+1}^{n} G_{i j}^{T T} T_{j}^{k}
\end{aligned}
$$

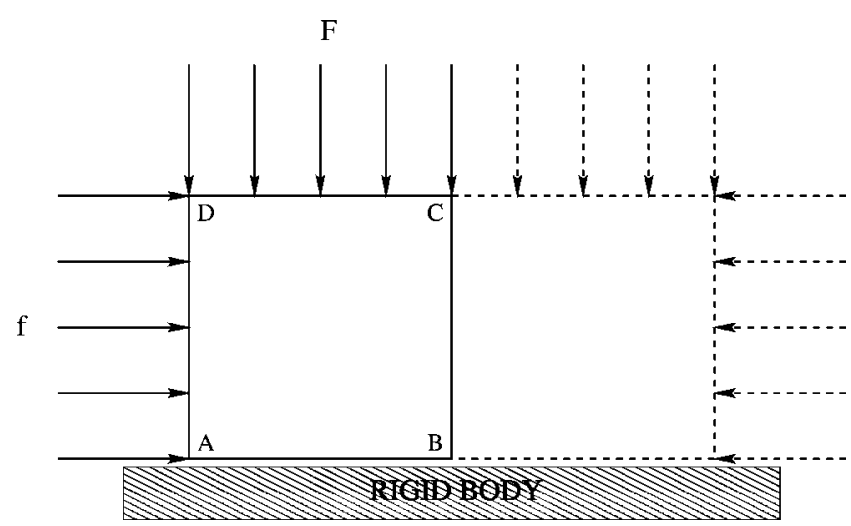

Fig. 1. The problem of the long bar in contact with a rigid plane.

$T_{i}^{k+1}=\frac{\beta}{G_{i i}^{T T}}$

$$
\begin{cases}\text { if } T_{i}^{k+(1 / 2)}>-\mu N^{k+1} & \text { then } T_{i}^{k+1}=-\mu N^{k+1} \\ \text { if } T_{i}^{k+(1 / 2)}<\mu N^{k+1} & \text { then } T_{i}^{k+1}=\mu N^{k+1} \\ \text { if }\left|T_{i}^{k+(1 / 2)}\right| \leq-\mu N^{k+1} & \text { then } T_{i}^{k+1}=T_{i}^{k+(1 / 2)}\end{cases}
$$

Usually, this algorithm is improved introducing a relaxation coefficient $\omega . N_{i}^{k+(1 / 2)}$ (resp. $T_{i}^{k+(1 / 2)}$ is replaced by $\omega N_{i}^{k+(1 / 2)}+(1-\omega) N_{i}^{k}\left(\operatorname{resp} . \omega T_{i}^{k+(1 / 2)}+(1-\omega) T_{i}^{k}\right)$ in the previous algorithm.

It is noted that, as classically, the stop criterion is defined on the relative variation of the contact and friction forces.

\section{Numerical results}

\subsection{The numerical example: a classical benchmark}

In order to test and validate the algorithm, we have chosen a benchmark studied by the group 'Validation of computer codes' of French Research Groupment 'Large Deformations and Damage' [10].

We work in plane strains and we consider a long bar with a rectangular section, with Young's modulus $E=130 \mathrm{GPa}$ and Poisson's ratio $\nu=0.2$. By symmetry reasons, we consider only a square section. The vertice lenght is equal to $40 \mathrm{~mm}$. The contact zone is the line $A B$ on Fig. 1.

The problem has been tested with different values of the friction coefficient $\mu$ and of the loading ( $f$ on $A D, F$ on $C D$ ).

Table 1

Behavior of the interface for different parameters

\begin{tabular}{lrllll}
\hline$\mu$ & $F$ & $f$ & $\begin{array}{l}\text { Gap } \sigma_{N}=\sigma_{T} \\
=0(\mathrm{~mm})\end{array}$ & $\begin{array}{l}\text { Sliding }\left|\sigma_{T}\right| \\
=f\left|\sigma_{N}\right|(\mathrm{mm})\end{array}$ & $\begin{array}{l}\text { Stick }\left|\sigma_{T}\right| \\
<f\left|\sigma_{N}\right|(\mathrm{mm})\end{array}$ \\
\hline 0.2 & -5 & 10 & 0 & 40 & 0 \\
0.2 & -15 & 10 & 0 & 23 & 17 \\
0.2 & -25 & 10 & 0 & 3 & 37 \\
1 & -5 & 10 & 3 & 18 & 19 \\
1 & -5 & 15 & 3 & 25 & 12 \\
\hline
\end{tabular}




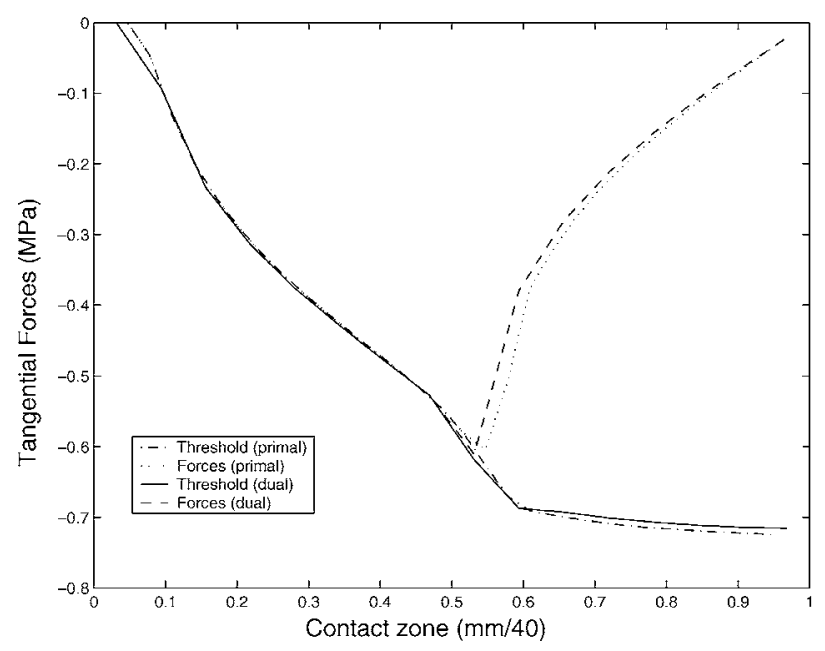

Fig. 2. Contact forces: primal and dual algorithms.

Variations of contact status are given in Table 1 for different parameters (the loadings are in $\mathrm{MPa}$ ).

\subsection{Agreement with primal formulation}

In this section, we compare the numerical results with thus obtained by the primal formulation [10] in order to analyse the precision of the algorithm. In Ref. [10], the problem is discretized by $P 1$ linear finite element (three nodes triangles) and the discrete problem is solved by a fixed point method coupled with a relaxation algorithm. The mesh is an unstructured one with 230 nodes ( 33 contact nodes). In our work, we have treated the same problem with a structured mesh with 289 nodes (17 contact nodes). The problem is discretized by bicubic Hermite elements [17] (four nodes quadrangles).

Fig. 2 shows that we obtain a good agreement between the two formulations in terms of contact forces. In Fig. 3, we observe the normal displacements for the two formulations

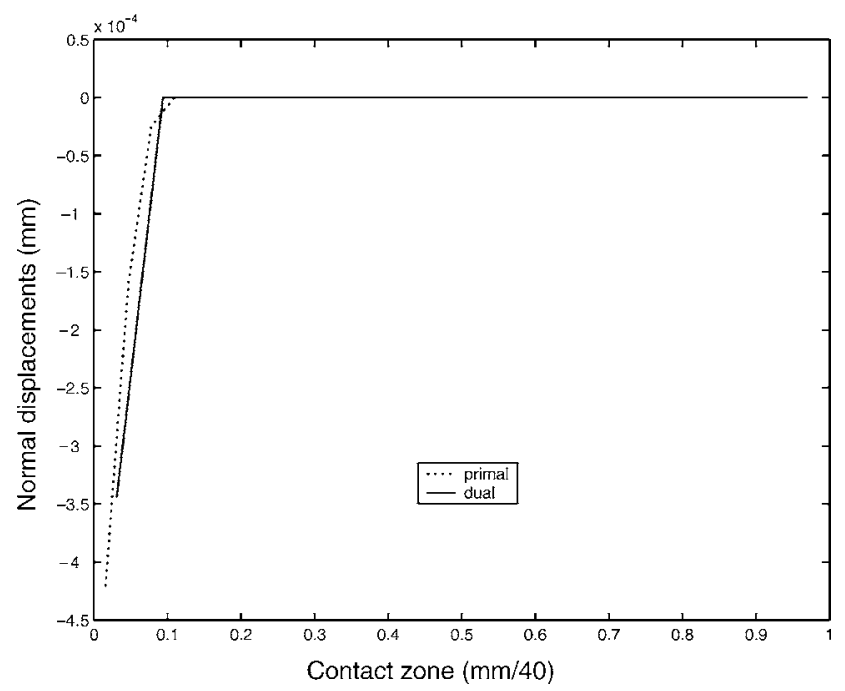

Fig. 3. Normal displacements on the contact zone: primal and dual algorithms.

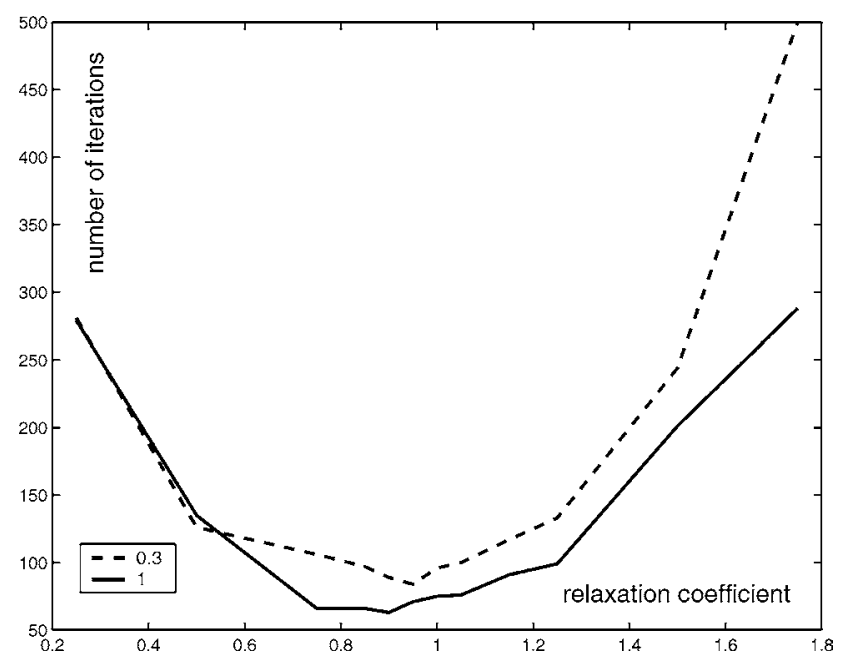

Fig. 4. Evolution of the relaxation parameter.

which could be an handicap for the development of our method to large slidings [18]. These figures correspond to the case $\mu=1, F=-5 \mathrm{MPa}$ and $f=15 \mathrm{MPa}$ (fifth line in Table 1). Note that D-PANA and D-GLT algorithms give the same solution. The low differences between the curves can be explained by the various approximations: P1 linear or bicubic Hermite elements,

- the various meshes: triangles with 230 nodes or quadrangles with 289 nodes,

- the computation of displacements and forces: by direct computation or by duality.

\subsection{Remarks on the algorithms behaviour}

In Fig. 4, the influence of the relaxation parameter $\omega$ on the convergence of the D-GLT algorithm is observed for two values of the friction coefficient (0.3 and 1.0). The optimal value of this coefficient is found to be close to 1 ; therefore, the search for an optimal $\omega$ needed in the primal algorithm

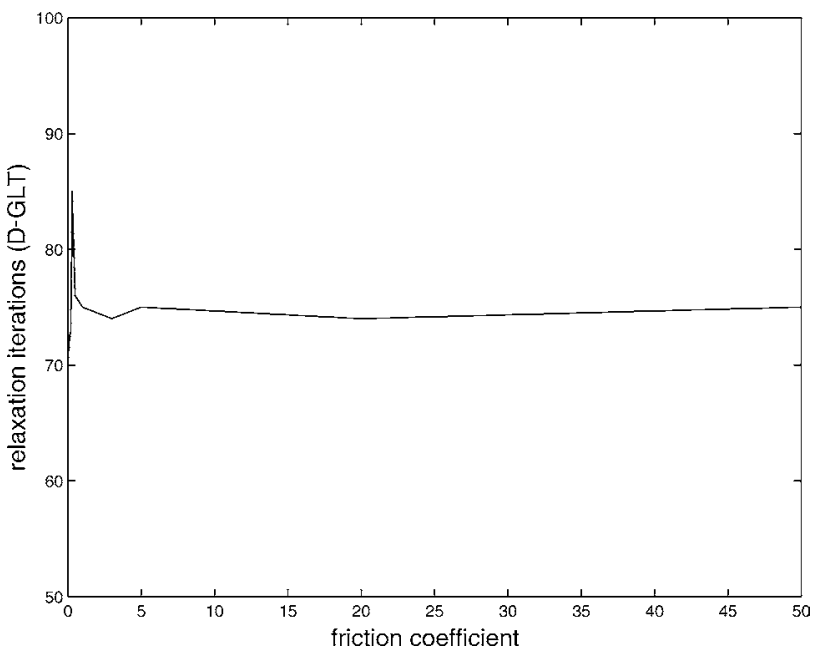

Fig. 5. Number of iterations versus friction coefficient. 
can be avoided. As an example, in [10], the optimal value of the relaxation coefficient is equal to 1.67 in the case presented above and the cost to obtain this coefficient is very high.

Fig. 5 shows the influence of the friction coefficient on the convergence for the D-GLT algorithm. This parameter has not a strong influence on the number of iterations (close to 75 in this case) contrary to more classical ones $[10,19,20]$.

\section{Conclusions}

In this paper, the formulation of frictional contact problems in terms of dual unknowns (stresses) was presented and solution methods of the problem issued from this formulation were investigated. A condensation on the initial contact area led to a discrete quasi-variational inequality. In our contribution, the latter was solved by means of two algorithms. These algorithms were implemented in a finite element software. Efficiency and robustness in two dimensions were shown and an excellent agreement with the numerical results supplied by primal formulations was obtained, both for stresses and displacements. Note that the development of this methodology in three dimensions leads to the 'facetization' of the Coulomb cone as in Ref. [13]. Other numerical examples with a more complex behaviour law were presented in Ref. [3].

\section{Acknowledgements}

This research was developed within the framework of Lagrange Laboratory, an European research group between CNRS, CNR, University of Rome 'Tor Vergata', University of Montpellier II, ENPC and LCPC.

\section{References}

[1] G. Duvaut, J.L. Lions, Inequalities in mechanics and physics, Springer Verlag, Berlin, 1976.

[2] Panagiotopoulos PD. A nonlinear programming approach to the unilateral and friction boundary value problem in the theory of elasticity. Ingenieur Archiv 1975;44:421-32.
[3] Bisegna P, Lebon F, Maceri F. The unilateral frictional contact of a piezoelectric body with a rigid support. In: Martins JMC, Marques MM, editors. Contact Mechanics International Symposium Proceedings of the Third International Conference, Dordrecht: Kluwer; 2002.

[4] Bisegna P, Lebon F, Maceri F. D-PANA : a convergent blockrelaxation solution method for the discretized dual formulation of the Signorini-Coulomb contact problem. Comptes Rendus Académie des Sciences, Série I 2001;333:1053-8.

[5] Lebon F, Raous M. Friction modelling of a bolted junction under internal pressure loading. Comput Struct 1992;43:925-33.

[6] Telega JJ. Topics on unilateral contact problems of elasticity and inelasticity. In: Moreau JJ, Panagiotopoulos PD, editors. Nonsmooth mechanics and applications. Berlin: Springer; 1988. p. 341-462.

[7] Kikuchi N, Oden JT. Contact problems in elasticity: a study of variational inequalities and finite element methods. SIAM, Philadelphia; 1988.

[8] Hlavacek I, Haslinger J, Necas J, Lovisek J. Solution of variational inequalities in mechanics. New York: Springer; 1988.

[9] Haslinger J, Dostal Z, Kucera R. On a splitting type algorithm for the numerical realization of contact problems with Coulomb friction. Comput Meth Appl Mech Engng 2002;191:2261-81.

[10] Raous M, Chabrand P, Lebon F. Numerical methods for solving unilateral contact problem with friction. J Theor Appl Mech 1988;7: $111-28$.

[11] Cocu M. Existence of solutions of Signorini problems with friction. Int J Engng Sci 1984;22:567-75.

[12] Mosco U. Implicit variational problems and quasivariational inequalities. Lecture notes in mathematics, Berlin: Springer; 1976.

[13] Klarbring A, Bjorkman G. The treatment of problems in contact mechanics by mathematical programming. J Theor Appl Mech 1988; 7:111-28.

[14] Capuzzo-Dolcetta I, Matzeu M. Duality for implicit variational problems and numerical applications. Numer Funct Anal Optim 1980; 2:231-65.

[15] Klarbring A, Mikelic A, Shillor M. On friction problems with normal compliance. Nonlinear Anal, Theory, Meth Appl 1989;13:935-55.

[16] Glowinski R, Lions JL, Trémolières R. Analyse numérique des inéquations variationnelles, Dunod, Paris; 1975.

[17] Maceri F, Bisegna P. The unilateral frictionless contact of a piezoelectric body with a rigid support. Math Comput Model 1998; 28:19-28.

[18] Cescotto S. On the numerical modelling of unilateral contact : primal, dual and mixed approached. Proceedings of the Eighth International Conference of Enhancement of Computing Methods for Engineering and Science, Shangai; 2001.

[19] Alart P, Lebon F. Numerical study of a stratified composite coupling homogenization and frictional contact. Math Comput Model 1998;28: $272-86$.

[20] Lebon F. Contact problems with friction: models and simulations. Simulat, Model, Theory Practice 2003;11:449-64. 